\title{
Quality of life in psoriatic arthritis
}

\section{Tania Gudu 1 , Laure Gossec 2}

Affiliations

1

Research Center of Rheumatic Diseases, Sf Maria Hospital, University of Medicine and Pharmacy Carol Davila , Bucharest , Romania.

- 2

Department of Rheumatology, Sorbonne Université, Institut Pierre Louis d'Epidémiologie et de Santé Publique; AP-HP, Pitié Salpêtrière Hospital, Paris, France.

Word count: 5217 excluding Abstract, Key issues, References and Figure/Table legends)

Keywords (alphabetical order 5-10): assessment, disease activity, fatigue, patientreported outcomes, psoriatic arthritis, psychological distress, quality of life

Conflict of interests: none for this work 
Structured abstract (200 words; maximum 200 words):

Introduction: Psoriatic arthritis (PsA) is a multi-organ chronic inflammatory disease which impacts patients both physically and psychologically. The highest priority for patients goes to pain relief, but also to ability to function and participate in social life, fatigue, and psychological distress.

Areas covered: In the present article, we will review current knowledge on impairments of health-related quality of life related to PsA, as well as patient priorities and patient-reported outcome measures such as the PsA Impact of Disease or the PsA Quality of Life questionnaires. The impact of PsA appears to be very broad, covering all aspects of life, i.e., activities and participation, physical and emotional aspects, but also domains such as fatigue, coping or sleep disturbance. Some of these aspects which are important for PsA patients I have been included in the recently updated PsA core domain set to be reported in clinical studies.

Conclusion: A better understanding of quality of life issues face by patients with PsA could improve patient-physician communication and ultimately, quality of care.

Expert commentary: QoL is altered in PsA due both to the physical impact, but also the psychological impact of this disease. Several scores are available to better assess these aspects of PsA. 
Body of the article

1. Introduction

According to the World Health Organization (WHO), quality of life (QoL) is defined as "an individual's perception of their position in life in the context of the culture and value systems in which they live and in relation to their goals, expectations, standards and concerns. It is a broad ranging concept affected in a complex way by the person's physical health, psychological state, personal beliefs, social relationships and their relationship to salient features of their environment" [1]. QoL as defined above is a multifaceted concept that usually includes subjective evaluations of both positive and negative aspects of life. Many terms are used when discussing the effects of diseases on functioning and sense of well-being, e.g., QoL, health status, impact of disease, or the more specific health-related QoL. In the present paper, we will refer to QoL. QoL is usually broken down into functioning, physical aspects (such as pain), psychological aspects (such as depression) and social impact of disease (such as being a burden to others) [2].

WHO has developed and endorsed a standard language and classification system for functioning and disability: the International Classification of Functioning, Disability and Health (ICF) [3]. In the ICF, functioning and disability are multidimensional. concepts. Both are umbrella terms for body function, body structures, and activities and participation. They denote the positive or neutral aspects (for functioning) or negative aspects (for disability) of the interaction between a person's health condition(s) and that individual's contextual factors 
(environmental and personal factors). The ICF has two parts. The ICF part 1 covers functioning and disability and includes the components body functions, body structures, and activities and participation. Part 2 covers contextual factors and includes the components environmental factors and personal factors. The ICF consists of multiple categories in each of these section [3].

It is particularly important to assess QoL in arthritis patients because the chronic debilitating nature of these diseases does not cause death, but has a substantial impact on QoL. Patients with inflammatory arthritis report higher impairment on everyday life in all its aspects: physical/functional, emotional and social compared to controls [4-11].

Psoriatic arthritis (PsA) is a chronic inflammatory arthritis, usually seronegative for rheumatoid factor, associated with psoriasis, with a prevalence of around $1 \%$ in the general population [12]. It is a multifaceted disease: PsA patients may present musculo-skeletal involvement (peripheral arthritis, dactylitis, enthesitis, spine inflammation), skin and nail disease or extra-articular manifestations (mainly uveitis and inflammatory bowel disease) [12].

PsA is a unique and distinctive disease because it sums up the effect of both arthritis and skin psoriasis, each with an important burden on patients' lives. PsA was shown to have a profound impact on QoL, with affected individuals experiencing chronic pain, limitations in physical functioning and work abilities, extreme fatigue and emotional and social impairment $[4-8,13,14]$. It is therefore very important to assess QoL in PsA. This has been recognized by including the HRQoL or QoL domain in the PsA Core Domain Set from work of the Group for 
Research and Assessment of Psoriasis and Psoriatic Arthritis (GRAPPA) and endorsed by the Outcome Measures in Rheumatology (OMERACT) group, both in 2006 [Gladman J Rheumatol. 2007] and 2016 [Orbai, et al, ARD 2017]. The Core Domain Set includes the domains that are mandatory to be measured in all PsA clinical trials and longitudinal observational studies. Impaired domains of QoL in PsA

In PsA the range of problems experienced by patients is broad and involves both physical and emotional health, but has also negative impact on daily activities, work and leisure activities, and social participation [14, 15]. We will review the alterations to QoL, by broad domains of health.

\subsection{Qualitative aspects of impact of PsA}

Although generic questionnaires and quantitative methodology can offer an objective view on the extent of the burden of the PsA, in particular by comparing across diseases, the most accurate method to evaluate the impact of the disease is to ask the patients directly. Qualitative methodology (such as focus groups or individual in-depth interviews) provide the opportunity to explore the perspective of those who experience the disease, i.e., the patient's perspective in detail. Such studies have yielded a wealth of information in rheumatoid arthritis (RA). In PsA however, there have been few published qualitative studies to date, although several are ongoing [16-21].

Qualitative methods have been included in the development of questionnaires or instruments [22-24], providing valuable information on patients' perspective on disease impact or QoL. In the elaboration of a questionnaire of 
disease impact, the PsA Impact of Disease (PsAID) [2],16 domains of health that were considered important by patients were identified (able 1). Furthermore, in a priority exercise, 474 PsA patients ranked these domains in the order of perceived importance: pain, fatigue, skin problems, work/ leisure activities and functional capacity were considered to be the most important (igure 1). Similarly, the development of the PsA QoL instrument (PsAQoL) [23] questionnaire yielded domains of health that could be categorized into four main experiences: reaction to diagnosis, life changes, adaptation and acceptance and concerns for the future. These included, as expected, pain or skin disease, but also other domains such as fatigue, coping, emotional and social problems

In a systematic review on patient involvement in outcome measures for PsA, Tillett et al [Tilleet, Curr Rheumatol Rep 2014] have shown that there has been little incorporation of the patient perspective in the development of outcomes measures and domains in PsA. However, the importance of including patient's perspective has been recognized and supported by both OMERACT [Boers, J Clin Epidemiol 2014] and European League Against Rheumatism (EULAR) [de Wit, ARD 2011] who strongly recommend the inclusion of patient research partners (PRPs) and other relevant stakeholders in all stages of the research process.

Efforts are made to increase involvement of PsA patients in research activities, e.g, core domains, treatment recommendations, etc. Specifically, qualitative research had a central role in the Core Domain Set development for PsA [Orbai, et al, ARD 2017]. In this process, 130 patients from 7 countries representing 5 continents worked in $\mathbf{2 4}$ focus groups in order to identify patienrelevant domains. This ensured capturing a broad content early on in the process of data collection and obtaining domains that were generalizable to people with PsA worldwide. The results of this study are of major importance since it 
demonstrated that patients' and physicians' priorities are different, therefore the inclusion of patient perspective brings additional, essential information. Based on these findings, adequate instruments or outcome measures are currently being selected for each core domain.

\section{$4 \mathrm{~L}[(\mathrm{r}$ (m tL[ dd s1}

The domains of impact identified through qualitative studies bring to light aspects that weren't previously reported as important in PsA, such as fatigue, social and sexual life, coping mechanisms, emotional problems such as anger, anxiety, fear, and embarrassment and shame due to appearance [15] (Figure 2). The perspective of patients is crucial when assessing QoL or impact of disease; corresponding instruments should be based on information derived directly from patients, ensuring that respondents find the instrument acceptable and relevant to their condition. This should be taken into consideration particularly in PsA where QoL instruments should reflect both rheumatic and dermatological impact on patients [16].

2.2. Reporting domains of impact in clinical studies and the Core Domain Set

Several years ago, the international groups OMERACT and GRAPPA decided through consensus on a Core Set of variables to be collected in clinical trials and observational longitudinal studies of PsA [ 0]. The PsA Core Domain Set consists of the 'inner circle' comprising domains that are mandatory to be measured in clinical trials and observational studies of PsA, 
and the 'outer circle' corresponding to domains which are not necessary to assess in PsA trials or domains which are still under evaluation (figure 4).

Over the last years, and based in particular on input from patient research partners, the Core Domain Set has been revisited. In a first step, a systematic literature review has shown that existing, frequently-used outcome measures have been devised with little or no involvement of patients [16]. Another study showed that none of these instruments captured the full experience of PsA from the perspective of patients [17]. Thereafter, patients and physicians worked together to identify domains that are important to patients and to update the PsA Core Set so that it also reflects the perspective of patients [11]. The input of patients played a key role in the process of developing the Domain Core Set: domains important for PsA patients were identified through an impressive large qualitative study, involving 24 focus groups of 130 patients from 7 countries representing 5 continents [Orbai, ARD 2017]. As stated above, this heterogeneity of patients involved ensured a broad variety of collected information, offering a high degree of generalizability.

Further on, the domains identified through the international focus groups and the systematic literature review were rated for importance by both patients and physicians in web-based surveys, followed by a face-to-face nominal group discussion including again PRPs and clinicians from around the world, resulting in the final list of domains considerend important to be measured in PsA studies [Orbai, ARD 2017]. The 2016 OMERACT-endorsed PsA Core Domain Set was hence developed based on qualitative research and 
patient research partner involvement at each step, giving validity to the resulting proposed domains [51].

In the updated core set, besides domains such as physical function, pain, health-related QoL (considered here as single entity) and patient global assessment which were considered essential already in its first version, awareness was raised for other domains important for patients. Thus, fatigue was recognized to be very important for PsA patients and consequently included in the inner circle; and emotional well-being and participation were added to the middle circle, as relevant domains of impact, but not always feasible to assess in all PsA studies (igure 4). These findings confirm the previously demonstrated broad life impact of PsA.

As stated above, the development of the 2016 PsA Core Domain Set also included a systematic review which identified domains and outcome measures used in PsA studies published between 2010 and 2015 [52]. According to this review, the domains important for patients that were most assessed were pain, which was included in most of the studies, followed by function, patient global and quality of life (table 3). Other domains such as fatigue and productivity, and to a lesser extent participation, coping or well-being were not so frequently reported (table 3). However, compared to results reported from a similar systematic literature review [47] on studies published in 2006-2010, there is clearly a growing trend towards reporting these domains. The area of life impact is currently least represented in all studies, but the new updated core set domains may help increasing this trend. 


\section{3.. Patient reported domains of impact}

The impact of PsA is broad, covering almost all aspects of life. Patients report impairment due to the disease in several domains that they consider relevants [Taylor, Curr Rheumatol Rep 2012; Gudu, J Rheumatol 2017; Orbai, ARD 2017]. The most frequent reported domains of impact are: activities and social participation, physical domains, emotional domains, fatigue, sleep, coping, intimacy and sexual life, and self-care. We will now review these in more detail.

\section{Activities and Social participation}

In PsA some of the most impaired aspects of QoL cover the area of activities (ability to work, leisure) and social participation (social relationships, intimacy, community participation) $[14,15,25]$ (figure 3 ).

In a study assessing the effect of PsA on 94 patients from five different countries according to the ICF, it was shown that the most impaired component was Activities and Participation [25]. Also, in a systematic review of studies assessing impact of PsA from the patient perspective [15], by linking concepts and domains of impact extracted from these studies to the ICF, it was demonstrated that in PsA the most affected category was again Activities and Participation (42.6\%). These results are of importance since they reflect the 
authentic patient perspective. On the contrary, in other rheumatic diseases the most affected components according to the ICF are related to body structures and body functions: $44.9 \%$ in RA [26] and $52.5 \%$ in axial spondyloarthritis [27]. This difference might be explained by the burden of both skin and arthritis and highlights once more the complexity of PsA.

PsA patients were found to have high levels of unemployment (20-50\%) and work disability (16-39\%), which are highly associated with longer disease duration, worse physical function, high joint count, low educational level, female gender, erosive disease and manual work [28]. In a study on 236 PsA patients in work from across the United Kingdom [29], reduced effectiveness at work was associated with measures of disease activity, whereas unemployment was associated with employer factors, age and disease duration. Other studies also demonstrated that work productivity as evaluated by the Work Limitations Questionnaire was decreased by $4.3-6.7 \%$ in patients with PsA compared with benchmark employees without limitations [30, 31].

In qualitative studies, domains related to activities and participation are frequently reported [15]: ability to work/volunteer, social participation (90.9\% of respondents each), leisure activities, family and intimacy (63.6\% each). For example, in one qualitative study, regarding the effect on leisure activities and participation, patients reported refusing invitations to social events or not completely enjoying the occasion:

"Sometimes, I am so tired that I don't go out, I stay at home" (R2, man, 67); 
"I was invited for a wedding, I didn't dance a lot and it was a problem for me" (R1, woman, 39) [20].

\section{Physical aspects}

PsA patients report high levels of pain and marked functional disability [7, 8, 13], which further impacts daily activities and work productivity [14] (figure 3). Physical disability is mostly due to the musculoskeletal component, since PsA patients suffer from arthritis, dactylitis, enthesitis, and sometimes axial involvement which results in pain, discomfort and reduced mobility. This component of the disease may in time lead to erosions and structural damage, with permanent disability [32]. However, skin disease can also cause pain and discomfort through itching, irritation, redness, skin soreness, or bleeding from psoriatic lesions.

In qualitative studies, the ICF components corresponding to physical aspects, i.e., Body structures and Body functions are also well represented (39.7\%) [15]. Domains such as Pain, Skin problems, Body Image (63.6\%) and Joint problems (45.4\%) are frequently reported [15].

Pain is an important symptom for PsA patients. In a priority exercise in which 474 PsA patients from 13 countries ranked these domains in the order of importance [22], pain was ranked as the most important domain of impact (figure 1). Skin problems and functional capacity were also highly ranked by these patients (figure 1). In another prioritization study on 59 PsA patients [33], the 
physical domain was considered to be the most important aspect of disease impact. Some of the differences observed may be explained by cultural background and/or disease severity [34].

Emotional aspects

Emotional and psychological symptoms are frequent and important in PsA (figure 3). Indeed, "emotional well-being" was selected by patients as important in the Core Domain Set development process [orbai], and was considered as a separate core outcome, but it was not included in the inner circle due to lack of instruments to specifically evaluate emotional well-being. Patients with PsA have a high prevalence of anxiety and depression symptoms, ranging from $7.9 \%$ to 36.6 [35-39]. The rate of both anxiety and depression was shown to be higher in PsA patients (36.6\% and $22.2 \%$, respectively) compared to those with psoriasis (24.4\% and $9.6 \%)$ [39]. Also, compared to RA patients $(21.7 \%$ of whom are depressed), the prevalence of moderate to severe levels of depressive symptoms is higher in PsA patients (25.1\%), especially in those with PsA with polyarthritis (36.7\%) [38]. Depression and anxiety are associated with various factors, both patient and disease related: unemployment status, female gender, actively inflamed joints, disability, pain and fatigue $[35,39]$. However, there is no association with treatment and interestingly, nor with skin psoriasis extent: anxiety and depression were shown to be independent of the Psoriasis Area and Severity Index [39, 40], explaining why patients with PsA have more anxiety and depression than those with psoriasis alone and confirming that patients' perceptions is more important than the actual objective severity of skin disease 
$[39,41]$. Assessment of depression and anxiety in PsA is important since they are associated with a poor QoL e [38] and may reduce likelihood of joint remission $[38,42]$ and contribute to poor treatment adherence [43].

Anxiety and depression are not the only psychological aspects that are frequent or important to patients with PsA (table 2). Data from qualitative studies or studies using some qualitative methodology show that PsA patients experience a broad variety of emotional feelings: anxiety, fear, uncertainty, concerns for the future, sadness, anger, depression, loss of motivation to take part in, and reduced pleasure gained from, activities, embarrassment and/or shame due to appearance, feeling rejected, concentration difficulties, frustration, being marked by visibility, preoccupation with the illness and grieving over loss of previous lifestyle $[17,20,22-24,33,44]$. Emotional problems are of high importance for patients suffering from PsA: $63 \%$ of patients have ranked emotional health as second most important domain of impact out of 8 domains [33]. Also, in a priority exercise of PsA patients, several domains related to emotional functions were ranked as being important for patients, among other 12 domains of impact: anxiety, embarrassment and/or shame, depression [22]. Moreover, when linking concepts and domains of impact extracted from 11 studies assessing impact of PsA from patient perspective to the ICF [15], the most frequently cited ICF category was b152 Emotional functions and was linked to a very wide range of concepts: anxiety, fear, uncertainty, sadness, depression, lack of motivation, irritation, frustration, anger etc, reflecting the high psychological burden of PsA. 


\section{Fatigue}

Fatigue is an important symptom in PsA (figure 3). Levels of fatigue in PsA are elevated: in a large cross-sectional cohort almost $50 \%$ of PsA patients reported moderate fatigue and $29 \%$ experienced severe fatigue [45] The importance attributed to fatigue is also high: in a priority exercise of 474 patients with PsA from 13 countries [46], patients ranked fatigue as the second most important domain after pain and before skin problems (figure 1). Data regarding causes of fatigue in PsA are sparse [45, 47, 48]: fatigue is reported to be mostly related to physical disability, pain and psychological distress in PsA. Recently, it was shown in a study of 246 patients with PsA from 13 countries [46] that high fatigue was explained mainly by disease-related factors (current skin psoriasis, tender joint count and enthesitis), but also patient-related characteristics (years of education and female gender), indicating that fatigue in PsA is multifactorial, but may be more strongly related to the disease process in PsA than in RA. However, another study evaluating fatigue in 101 PsA patients [49] showed that it might be more related to the emotional and social aspects of the disease than to joint inflammatory aspects, suggesting that the disease's visibility is the most disturbing aspect for the patient and that "skin pain" is more intense than the joint pain.

In qualitative studies, fatigue is frequently cited [15] and has a complex meaning to patients, encompassing both physical and mental aspects $[15,17,20]$. In the qualitative study preceding the development of the GRAPPA/OMERACT updated PsA Core Domain Set [Orbai, ARD 2017] fatigue was considered a critical 
element of life impact by PsA patients, along with participation and emotional well-being, i.e., more than $70 \%$ of patients reported fatigue as an important domain of impact. The recognition of the importance of fatigue translated consequently into including it in the inner circle of the core domain set, making it mandatory to evaluate in all PsA studies [Orbai, ARD 2017].

\section{Other domains of health}

There are other domains of impact that are important for PsA patients and that cannot be included in the categories above, as they comprise physical, emotional/ psychological and social aspects at the same time (figure 3 ). These domains of impact although not frequently assessed in trials, are frequently reported by patients in studies with qualitative methodology: fatigue (see above), sleep $[17,22,23,33$,$] , coping [20,22,23$, intimacy and sexual life $[17,20,22,33]$ or self-care $[17,33]$.

s ) [ eAtedn4 na[e[e rts1 1 h $) \mid$ sn se .t52 ((.m7

2. QoL in PsA compared to psoriasis and other rheumatic diseases

PsA has been shown to be a heterogeneous, disabling disease with a major impact on patients' lives [7, 8, 13]. Patients with PsA experience a reduced QoL compared to the general population, more specifically they report significantly 
lower scores on the physical functioning, pain, role limitations and general health perceptions [53].

PsA patients report higher impact on QoL than patients with psoriasis only [54-56]. These results are of major importance since high impairment of QoL in psoriasis patients, specifically on psycho-social domains of impact was already demonstrated [57-61. Compared to patients with psoriasis without arthritis, PsA patients have reported worse scores on multiple generic questionnaires assessing quality of life, showing a higher burden on domains of impact such as functional status, fatigue, and QoL in general but not mental health or effect of skin disease [54]. These results suggest that the burden of joint disease adds up to the diminished QoL caused by the skin disease. However, there are some conflicting results $[53,62]$. In a study comparing QoL and functional status of psoriasis and PsA patients with normal individuals [53], both groups had worse quality of life than healthy individuals. Although PsA patients had a worse functional status than the ones without arthritis, QoL was found to be similar between them. Nonetheless, the authors did not use a generic instrument to assess QoL, but a PsA-specific questionnaire, i.e., the PsAQoL [23].

Compared to other rheumatic diseases, i.e., RA or axial spondyloarthritis, although patients with RA had more destructive arthritis, with a higher impact on disability and pain, interestingly QoL was similarly altered in PsA $[7,8,11]$. In a study that compared QoL in RA, PsA and psoriasis assessed by the generic instrument Short Form 36 (SF-36) and quantified using spydergrams [13], patients demonstrated different patterns of QoL profiles across groups (figure 5): patients 
with arthritis (either RA or PsA) reported high impact on physical function, pain and fatigue; in contrast, psoriasis patients had higher impact on mental domains such as social functioning. While the physical health domains were much more altered in PsA than in psoriasis, mental health scores were similar, with the exception of fatigue which was substantially lower in PsA, too. These results suggest that much of the negative mental impact in PsA is due to the skin disease.

Another important point relates to comorbidities in PsA. Patients with PsA present with more comorbidities than patients with other inflammatory rheumatic diseases, at the group level [Shah, Sobchak]. Some frequent comorbidities include obesity, metabolic syndrome, cardiovascular events, depression and fibromyalgia. These comorbidities negatively impact patients' QoL.[Husted]

The comparison of PsA versus RA and psoriasis regarding impact and quality of life is of major importance since it brings new perspectives in understanding how the two components of PsA impact patients' lives.

3. Instruments used to measure QoL in PsA: patient reported outcomes

Measuring health-related QoL in PsA can be performed with instruments that reflect the patient's perspective on the disease impact on QoL, the so-called patient reported outcomes (PROs). They are particularly important since they capture the information coming directly from patients and bring additional valuable information, ensuring a more 
have strong points from a psychometric point of view: they reflect the patient's perspective, i.e., a unique information that cannot be collected from a physician; they have good psychometric properties, i.e., face and construct validity, reliability [63-65]; high feasibility: usually costless, noninvasive, not painful, not requiring costly equipment [66 ] and some of them are cross-culturally validated [64]; and finally, sensitive to change [67].

In PsA there are several PROs used to measure the quality of life: generic and disease-specific, each with both advantages and disadvantages. These PROs have recently been reviewed in detail [Hojgaard].

\section{Generic PROs for QoL}

Generic questionnaires, i.e., questionnaires not developed specifically for PsA have several strong points: experience of use, wide validation in other diseases, and the possibility of comparing across diseases.

The Medical Outcomes Study SF36 is a generic questionnaire to measure QoL [68] and comprises 2 main components: physical health (PCS) and mental health (MCS) and 8 subdomains: Physical functioning (PF), role limitations due to physical problems (RP), bodily pain (BP), general health $(\mathrm{GH})$, vitality $(\mathrm{VT})$, social functioning $(\mathrm{SF})$, role limitations due to emotional problems (RE) and mental problems $(\mathrm{MH})$. The SF-36 has been validated in PsA [69; Leung, Rheumatology 2010] and is often used in clinical trials to assess the domain of health-related quality of life in PsA populations 
[47]. Minimally Clinical Important Differences (MCID) have been established for the SF-36 summary scores and are different for improvement and deterioration: 5.0 points and $-2,5$ points, respectively. However, the SF-36 has several disadvantages: it is copyrighted which may limit its use and has a difficult scoring algorithm, making it not feasible outside of clinical trial settings [69].

The EuroQol 5 domain (EQ5D) is a generic instrument providing a measure on overall health status [70]. It consists of two parts: a questionnaire and a rating scale. The questionnaire includes five dimensions of health: mobility, self-care, usual activities, pain/ discomfort, anxiety/ depression. The visual analog scale assesses self-rated health with end points labeled "Best imaginable health" and "Worst imaginable health." The EQ5D has shown discrimination and responsiveness in PsA trials [71].

The Dermatological Life Quality Index (DLQI) is a psoriasis 10-item questionnaire assessing the effect of psoriasis on daily activities and level of disability [72] The DLQI questions are grouped into 6 subcategories: symptoms and feelings, daily activities, leisure, work/school, personal relationships and treatment. The MCID for the DLQI has not been established for PsA, but in psoriasis patients it has been estimated as a 5point improvement [73].

\section{Disease-specific PROs for QoL}

Generic questionnaire may be inadequate when evaluating the impact of the disease from the patient's perspective, as they do not capture the duality of the 
disease, i.e. rheumatic and dermatological. In contrast, PsA-specific instruments may provide more insight into the specific areas of life impaired by PsA.

There are very few questionnaires which have been developed specifically for PsA. In a systematic literature review endorsed by GRAPPA/ OMERACT of PROs in PsA [74] ten PROs were identified; some of them were versions of the same instrument, e.g., shorter versions or touch-versions. Of these only three had some evidence on both reliability and validity: the PsAID questionnaire, the PsAQoL and the VITACORA-19 (Spanish and Italian versions).

\section{The PsAID questionnaire}

The (EULAR) recently developed the PSAID questionnaire, a multi-dimensional patient-reported questionnaire to assess the impact of PsA from the patient's perspective (table 4) [22, 75, 76]. Compared to existing instruments, the PsAID-score is unique because it has been developed with the active involvement of 11 patient research partners from 11 European countries and then on a prioritization exercise on 139 PsA patients from across Europe. It is therefore fully based on the patient perspective of the illness [75]. The validation study was performed in 13 countries (474 patients). The measures performed well: high reliability (intra-class correlation coefficient, $0.95,95 \%$ confidence interval $0.92-0.96$ ) and high sensitivity to change (standardized response mean 0.90) [22].

There are two versions of the PsAID: the 12-item version (PsAID12) recommended for clinical practice which covers domains such as pain, skin problems, fatigue, work and/or leisure activities, functional capacity, discomfort, 
sleep disturbance, anxiety/fear and uncertainty, coping, embarrassment and/or shame, social participation and depression; and the 9-item version (PsAID9) which is recommended for clinical research and which is shorter and does not contain the last three items: embarrassment and/or shame, social participation and depression (table 1). The PsAID questionnaires are available online free of charge with their many available translations (table 4) [76].

It has also been validated in other cohorts [77, 78], including a touch-screen version [79]. A score below 4 out of 10 is considered a patient-acceptable status [76], a change of 3 or more points is considered a relevant absolute change [76] and 1.41 is the minimal detectable change [78].

Although recent, the PsAID has the advantage of being patient-derived with very active involvement of patient research partners at several levels throughout [75] and it is a short and simple questionnaire with good psychometric properties, suitable for use in both research and clinical settings.

\section{The PsAQoL questionnaire}

The PsAQoL [23] assesses QoL defined as the extent to which needs are fulfilled and reflects impact from the perspective of the patient, since it was derived from unstructured, qualitative interviews conducted with patients with PsA. It includes 20 items consisting as far as possible of wording taken from the transcripts and covers various domains of impact such as physical problems, fatigue, emotional and social problems (table 1). It has good item fit and excellent psychometric properties: internal consistency 0.91 , test-retest reliability 0.89 and 
good external construct validity [23]. Assessment of sensitivity and responsiveness to change demonstrated significant change at 3 and 6 months after change of disease modifying therapy ( $p<0.01$ and $p<0.05$, respectively) [80], with large standardized response mean at 3 months $(0.71)$ and small at 6 months (0.41). The PsAQoL has been translated and validated in several languages [ 4]: Dutch, Swedish, Chinese and English for Singapore, Greekand Portuguese [64].

The PsAQoL has many strong points:, it is derived directly from qualitative interviews, has good psychometric and feasibility properties, making it suitable for use in both research and clinical settings. However, it has been little used to date both in clinical and research settings, due to the fact that 2 is subject to copyright and has a high license fee to use.

Other scores

The VITACORA-19 questionnaire has been recently developed and appears to have good psychometric properties though further testing is needed.[24, Hojgaard].

Other PsA-specific PROs include te4, the Arthritis Impact Measurement (AIMS) 1 Global [83], the Psoriatic Arthritis Impact Profile (PAIP) [84], PSOdisk [ [85], the Combined Inflammatory Arthritis - QoL questionnaire (CIAQ-QoL) [86], and the Inverse Psoriasis Burden of Disease (the IBOD) [87]. We will not describe these questionnaires which have been little used to date.

\section{Summary}


In the present article, we have reviewed current knowledge on impairments of health-related quality of life related to PsA, as well as patient priorities and patient-reported outcome measures such as the PsA Impact of Disease or the PsA Quality of Life questionnaires. The impact of PsA appears to be very broad, covering all aspects of life, i.e., activities and participation, physical and emotional aspects, but also domains such as fatigue, coping or sleep disturbance. Some of these aspects which are important for PsA patients have been included in the recently updated PsA core domain set to be reported in clinical studies.

\section{Conclusion}

| PsA has profound impact on patients' QoL, due to both physical aspects of impact but also alterations in psychological domains, and functioning/social consequences of the disease. Causality of changes in QoL in PsA are potentially complex and multiple, with an overlap between the inflammatory process of PsA, other direct effects of PsA, comorbidities or contextual factors [Leung].

Better assessment of QoL issues should contribute to better quality of care. 
Expert commentary:

Although PsA is sometimes considered a benign disease, the signs and symptoms of patients with PsA are highly variable since the disease can include for example, very limited or quite severe skin involvement, and very limited or quite severe joint involvement. These varied involvements alter patients' QoL. Recent studies have indicated that QoL is altered in PsA to similar levels as in other chronic diseases including RA. One aspect which is important to note is that the psychological impact of PsA is very important, the disease being often associated to psychological distress and low self-esteem. Also, PsA patients report a major impairment in social participation and in performing various activities. Therefore, scores and questionnaires used to assess QoL-related aspects in PsA need to cover a broad variety of domains: physical, emotional, social etc.

The outcome measures used in PsA studies to assess QoL do not always reflect patients' views and perceptions of disease impact. At the moment in the PsA literature there is a lack of qualitative studies that could provide the opportunity to explore the perspective of patients in detail. Still, over the last years, several studies have been performed to better understand patientperceived impact of PsA and questionnaires which perform well, are available to fully assess patients with this disease. It would be of particular interest to use qualitative methodology in order to deepen the knowledge on impact of PsA on domains that are not so well studied but are of high importance for PsA patients, 
e.g., social participation, family and intimate relationships, work, emotional problems, fatigue or coping.

Recently, the Core Domain Set (a consensus list of domains of health which should be assessed in trials and longitudinal studies) has been updated. Several patient-reported domains have been included in the Core Domain Set. Fatigue and HRQoL are considered as core domains, whereas other aspects of HRQoL like emotional well-being or participation are considered as separate outcomes needing further research. This indicates the growing importance given to better assessing patient priorities in PsA. However, it has not yet been determined what are the best tools or questionnaires to assess fully patients with PsA;. The GRAPPA/OMERACT group is currently developing a Core Instrument Set which should facilitate standardization of assessment of PsA. It will be interesting to see if the domains of health in the Core Domain Set are indeed assessed in future studies, and if so, this will be an opportunity to further our knowledge regarding QoL issues. Some important questions which need further clarification include causality (i.e., link between alterations in QoL and the disease process itself, versus other non-disease related issues such as work or family problems), and responsiveness of QoL outcomes after efficacious drugs (such as disease-modifying drugs) are given. If some aspects of QoL are found to be only weakly linked to the inflammatory process, it should be considered to propose interventions to these patients which are not centered around disease activity, for example, psychological support or non-pharmacological measures. 
Currently though, such support may be difficult to organize in many healthcare systems.

In conclusion, although there is a growing interest in assessing how QoL is impaired in PsA, more studies are needed in order to obtain a broad and accurate image of PsA impact and to understand how this impact should be taken into account, in particular in the context of shared decision-making between patients and physicians. 
Five-year review

Assessment of patients' QoL is and will continue to be of interest in rheumatology since it is the final objective of treatment. There is a growing interest in the direct evaluation of patient's perspective in rheumatology by using PROs in trials, observational studies and routine clinical practice [88-92]. This interest coincides with a global trend for more 'patient-centred care', for better shared decision-making and also for actively engaging with patients in the context of scientific research [93]. This principle has recently been applied in the field of PsA [75]. However, there is a paucity of qualitative data on impact of PsA compared to other diseases, e.g., RA.

The recent growing interest in patient's perspective in PsA will surely continue to increase in the next 5 years. Development of instruments and PROs is no longer possible without putting the patient's opinion in the inner core. In the years to come, clinical trials and observational studies will include besides "classical" outcomes, some domains such as social participation, emotional wellbeing or coping. Another challenge of PsA studies that will probably be improved in the next 5 years is to find appropriate instruments that can accurately reflect the patients' view on these domains of interest.

Some important questions which need further clarification include causality (i.e., link between alterations in QoL and the disease process itself, versus other non-disease related issues such as work or family problems), and responsiveness of QoL outcomes after efficacious drugs (such as diseasemodifying drugs) are given. Finally, the role of the assessment of QoL in the 
context of shared decision-making between patients and physicians will be explored over the coming years.

\section{Key issues}

1. Psoriatic arthritis (PsA) is a multifaceted disease, including variable associations of musculo-skeletal involvement (peripheral arthritis, dactylitis, enthesitis, spine inflammation), skin and nail disease or extra-articular manifestations.

2. Qol is profoundly altered in PsA, due to both physical aspects of impact but also alterations in psychological domains, and functioning/social consequences of the disease.

3. The alterations to QoL appear to be due both to the arthritis/ rheumatological component, and to the psoriasis/skin component.

4. Functional and social impairment in PsA patients have a high impact on a specific category of QoL, called Activities and Participation.

5. dnsPhysical domains of health and in particular pain are cited as patient priorities.

6. Fatigue is a key issue for patients though its causality is multifactorial.

7. The Core Set of domains to assess to trials and longitudinal studies, has been recently updated and now includes several patient-reported outcomes reflecting QoL.

8. Several scores are available to assess QoL in PsA: some are more adapted to trials, whereas others are promising for patient care. 
9. Better assessment of QoL issues, i.e., developing a Core Outcome Measurement Set for assessment of Core Domains should contribute to better quality of care in PsA.

\section{References}

1. WHOQOL Group. Development of the WHOQOL: Rational and current status. Int. J Mental Health 1994;23: 24-56

2. World Health Organization. WHOQOL-BREF introduction, administration, scoring and generic version of the assessment. Geneva: World Health Organization; 1996

3. World Health Organization. International classification of functioning, disability and health (ICF). [Internet. Accessed December 2, 2017] Available from: www.who.int/ classifications/icf/en/

4. Strand V, Singh JA. Newer biological agents in rheumatoid arthritis: impact on health-related quality of life and productivity. Drugs $2010 ; 70: 121-45$

5. Singh JA, Strand V. Spondyloarthritis is associated with poor function and physical health-related quality of life. J Rheumatol 2009;36:1012-1020

6. Sokoll KB, Helliwell PS. Comparison of disability and quality of life in rheumatoid and psoriatic arthritis. J Rheumatol 2001;28:1842-1846

7. Husted JA, Gladman DD, Farewell VT, et al. Health-related quality of life of patients with psoriatic arthritis: a comparison with patients with rheumatoid arthritis. Arthritis Rheum 2001;45:151-158 
8. Borman P, Toy GG, Babaoglu S, et al. A comparative evaluation of quality of life and life satisfaction in patients with psoriatic and rheumatoid arthritis. Clin Rheumatol 2007;26:330-334

9. Ovayolu N, Ovayolu O, Karadag G. Health-related quality of life in ankylosing spondylitis, fibromyalgia syndrome, and rheumatoid arthritis: a comparison with a selected sample of healthy individuals. Clin Rheumatol. 2011;30:655-664

10. Salaffi F, Sarzi-Puttini P, Girolimetti R, et al. Health-related quality of life in fibromyalgia patients: a comparison with rheumatoid arthritis patients and the general population using the SF-36 health survey. Clin Exp Rheumatol. 2009;27(5 Suppl 56):S67-74

11. Salaffi F, Carotti M, Gasparini S, et al. The health related quality of life in rheumatoid arthritis, ankylosing spondylitis, and psoriatic arthritis: a comparison with a selected sample of healthy people. Health Qual Life Outcome. 2009;7:25

12. Gladman DD, Antoni C, Mease P, et al. Psoriatic arthritis: epidemiology, clinical features, course, and outcome. Ann Rheum Dis. 2005;64 Suppl 2:ii14-7

13. Strand V, Sharp V, Koenig AS, et al. Comparison of health-related quality of life in rheumatoid arthritis, psoriatic arthritis and psoriasis and effects of etanercept treatment. Ann Rheum Dis 2012;71:1143-1150

*Study that compared QoL in rheumatoid arthritis, PsA and psoriasis, demonstrating different patterns of QoL profiles across these diseases, and bringing new perspectives in understanding how the two components of PsA (arthritis and skin disease) impact patients' lives 
14. Taylor WJ. Impact of psoriatic arthritis on the patient: through the lens of the WHO International Classification of Functioning, Health, and Disability. Curr Rheumatol Rep 2012;14:369-374

15. Gudu T, Kiltz U, de Wit M, et al. Mapping the Effect of Psoriatic Arthritis Using the International Classification of Functioning, Disability and Health. J Rheumatol. $2017 ; 44: 193-200$

** Article that reviewed qualitative PsA publications and linked them to the ICF, showing that PsA widely affects all aspects of patients' lives, in particular aspects related to activities and participation

16. Tillett W, Adebajo A, Brooke M, et al. Patient involvement in outcome measures for psoriatic arthritis. Curr Rheumatol Rep 2014;16:418.

17. Stamm TA, Nell V, Mathis $M$, et al. Concepts important to patients with psoriatic arthritis are not adequately covered by standard measures of functioning. Arthritis Rheum 2007;57:487-494

** Qualitative study on PsA patients' problems in daily functioning demonstrating that there are several concepts addressing participation that are currently not covered in the instruments assessing functioning in PsA.

18. Moverley AR, Vinall-Collier KA, Helliwell PS. It's not just the joints, it's the whole thing: qualitative analysis of patients' experience of flare in psoriatic arthritis. Rheumatology 2015;54:1448-1153

19. Dures $\mathrm{E}_{2}$ Hewlett $\mathrm{S}$, Lord J, et al. Important Treatment Outcomes for Patients with Psoriatic Arthritis: A Multisite Qualitative Study. Patient. 2017;10:455-462 
20. Szentpetery A, Johnson MA, Ritchlin CT. GRAPPA Trainees Symposium 2013: a report from the GRAPPA 2013 Annual Meeting. J Rheumatol. 2014;41:1200-1205

21. Gossec L. Remission and Flare in Psoriatic Arthritis: a Prospective 6-month Study With a Double Perspective (ReFlaP) (2017). Retrieved from https://clinicaltrials.gov/ ct2/show/NCT03119805?term =NCT03119805\&rank=1 (Identification No NCT03119805 )

22. Boers M, Kirwan JR, Wells G, et al. Developing core outcome measurement sets for clinical trials: OMERACT filter 2.0. J Clin Epidemiol 2014;67:745-53.

23. de Wit MP, Berlo SE, Aanerud GJ, et al. European League Against Rheumatism recommendations for the inclusion of patient representatives in scientific projects. Ann Rheum Dis 2011;70:722-6

24. Gossec L, de Wit M, Kiltz U, et al. A patient-derived and patient-reported outcome measure for assessing psoriatic arthritis: elaboration and preliminary validation of the Psoriatic Arthritis Impact of Disease (PsAID) questionnaire, a 13-country EULAR initiative. Ann Rheum Dis 2014;73: 1012-1019

** Patient-derived instrument (questionnaire) to assess PsA impact, applicable in daily practice, with active involvement of patients at several levels throughout.

25. McKenna SP, Doward LC, Whalley D, et al. Development of the PsAQoL: a quality of life instrument specific to psoriatic arthritis. Ann Rheum Dis 2004;63:162-169

26. Torre-Alonso JC, Gratacos J, Rey-Rey JS et al. Development and validation of a new instrument to measure health-related quality of life in patients with psoriatic arthritis: the VITACORA-19. J Rheumatol 2014;41:2008-2017 
27. Taylor WJ, Mease PJ, Adebajo A, et al. Effect of psoriatic arthritis according to the affected categories of the international classification of functioning, disability and health. J Rheumatol. 2010;3:1885-1891

28. Stucki G, Cieza A, Geyh S, et al. ICF Core Sets for rheumatoid arthritis. J Rehabil Med 2004;44 Suppl:87-93

29. Boonen A, Braun J, van der Horst Bruinsma IE, et al. ASAS/WHO ICF Core Sets for ankylosing spondylitis (AS): how to classify the impact of AS on functioning and health. Ann Rheum Dis 2010;69:102-107

30. Tillett $\mathrm{W}$, de-Vries $\mathrm{C}, \mathrm{McHughNJ}$. Work disability in psoriatic arthritis: a systematic review. Rheumatology (Oxford) 2012;51:275-283

31. Tillett W, Shaddick G, Askari A, et aş. Factors influencing work disability in psoriatic arthritis: first results from a large UK multicentre study. Rheumatology (Oxford). $2015 ; 54: 157-162$

32. Kennedy M, Papneja A, Thavaneswaran A, et al. Prevalence and predictors of reduced work productivity in patients with psoriatic arthritis. Clin Exp Rheumatol 2014;32:342-348

33. Walsh JA, McFadden ML, Morgan MD, et al. Work productivity loss and fatigue in psoriatic arthritis. J Rheumatol 2014;41:1670-1674.

34. Siannis F, Farewell VT, Cook RJ, et al. Clinical and radiological damage in psoriatic arthritis. Ann Rheum Dis. 2006;65:478-481

35. Hu SW, Holt EW, Husni ME, et al. Willingness-to-pay stated preferences for 8 health-related quality-of-life domains in psoriatic arthritis: a pilot study. Semin Arthritis Rheum 2010;39:384-397 
36. Putrik $\mathrm{P}$, Ramiro $\mathrm{S}$, Hifinger $\mathrm{M}$, et al. In wealthier countries, patients perceive worse impact of the disease although they have lower objectively assessed disease activity: results from the cross-sectional COMORA study. Ann Rheum Dis. $2016 ; 75: 715-720$

37. Freire M, Rodriguez J, Moller I, et al. Prevalence of symptoms of anxiety and depression in patients with psoriatic arthritis attending rheumatology clinics [Spanish]. Reumatol Clin 2011;7:20-26

38. Khraishi M, MacDonald D, Rampakakis E, et al. Prevalence of patient-reported comorbidities in early and established psoriatic arthritis cohorts. Clin Rheumatol $2011 ; 30: 877-885$

39. Husted JA, Thavaneswaran A, Chandran V, et al. Cardiovascular and other comorbidities in patients with psoriatic arthritis: a comparison with patients with psoriasis. Arthritis Care Res 2011;63:1729-1735

40. Kotsis K, Voulgari PV, Tsifetaki N, et al. Anxiety and depressive symptoms and illness perceptions in psoriatic arthritis and associations with physical health-related quality of life. Arthritis Care Res 2012;64:1593-1601

41. McDonough $\mathrm{E}_{\mathrm{i}}$, Ayearst $\mathrm{R}$, Eder $\mathrm{L}$, et al. Depression and anxiety in psoriatic disease: prevalence and associated factors. J Rheumatol. 2014;41:887-896

42. Bandinelli F, Prignano F, Bonciani D, et al. Clinical and demographic factors influence on anxiety and depression in early psoriatic arthritis (ePsA). Clin Exp Rheumatol 2013;31:318-319

43. Schmitt J, Ford DE. Understanding the relationship between objective disease severity, psoriatic symptoms, illness-related stress, health-related quality of life and 
depressive symptoms in patients with psoriasis - a structural equations modeling approach. Gen Hosp Psychiatry 2007;29:134-140

44. Michelsen B, Kristianslund EK, Sexton J, et al. Do depression and anxiety reduce the likelihood of remission in rheumatoid arthritis and psoriatic arthritis? Data from the prospective multicentre NOR-DMARD study. Ann Rheum Dis. 2017;76:1906-1910

45. Betteridge $\mathrm{N}$, Boehncke $\mathrm{WH}$, Bundy $\mathrm{C}$, et al. Promoting patient-centred care in psoriatic arthritis: a multidisciplinary European perspective on improving the patient experience. J Eur Acad Dermatol Venereol 2016;30:576-585.

46. Uttjek M, Nygren L, Stenberg B, et al. Marked by visibility of psoriasis in everyday life. Qual Health Res 2007;17:364-372.

47. Husted JA, Tom BD, Schentag CT, et al. Occurrence and correlates of fatigue in psoriatic arthritis. Ann Rheum Dis 2009;68:1553-1558.

48. Gudu T, Etcheto A, de Wit M, et al. Fatigue in psoriatic arthritis - a cross-sectional study of 246 patients from 13 countries. Joint Bone Spine. 2016;83:439-443

49. Palominos PE, Gaujoux-Viala C, Fautrel B, et al. Clinical outcomes in psoriatic arthritis: A systematic literature review. Arthritis Care Res (Hoboken) 2012;64:397-406

50. Husted JA, Tom BD, Farewell VT, et al. Longitudinal analysis of fatigue in psoriatic arthritis. J Rheumatol 2010;37:1878-1884.

51. Carneiro C, Chaves M, Verardino G, et al. Evaluation of fatigue and its correlation with quality of life index, anxiety symptoms, depression and activity of disease in patients with psoriatic arthritis. Clin Cosmet Investig Dermatol. 2017;10:155-163 
52. Gladman DD, Mease PJ, Strand V, et al. Consensus on a core set of domains for psoriatic arthritis. J Rheumatol 2007;34:1167-1170

53. Orbai $A M$, de Wit $M$, Mease $P$, et al. International patient and physician consensus on a psoriatic arthritis core outcome set for clinical trials. Ann Rheum Dis. 2017; 76: $673-680$

** The 2016 updated PsA Core Set (a consensus list of domains of health that should be assessed in PsA studies). Several patient-reported domains, e.g., fatigue, emotional well-being, participation have been included in this list, indicating the growing importance given to better assessing patient priorities in PsA.

54. Kalyoncu U, Ogdie A, Campbell W, et al. Systematic literature review of domains assessed in psoriatic arthritis to inform the update of the psoriatic arthritis core domain set. RMD Open2016;2:e000217

* Systematic literature review on domains assessed in PsA studies showing an increasing trend towards reporting domains that are important for PsA patients: fatigue, productivity, and to a lesser extent participation, coping and well-being.

55. Tezel N, Yilmaz Tasdelen O, Bodur $\mathrm{H}$, et al. Is the health-related quality of life and functional status of patients with psoriatic arthritis worse than that of patients with psoriasis alone? Int J Rheum Dis. 2015;18:63-69

56. Rosen CF, Mussani F, Chandran V, et al. Patients with psoriatic arthritis have worse quality of life than those with psoriasis alone. Rheumatology (Oxford). 2012;51:571-576 
57. Lundberg L, Johannesson M, Silverdahl M, et al. Health-related quality of life in patients with psoriasis and atopic dermatitis measured with SF-36, DLQI and a subjective measure of disease activity. Acta Derm Venereol. 2000;80:430-434

58. Zachariae H, Zachariae R, Blomqvist K, et al. Quality of life and prevalence of arthritis reported by 5,795 members of the Nordic Psoriasis Associations. Data from the Nordic Quality of Life Study. Acta Derm Venereol. 2002;82:108-113

59. Krueger G, Koo J, Lebwohl M, et al. The impact of Ps on quality of life: results of a 1998 National Ps Foundation patient-membership survey. Arch Dermatol. $2001 ; 137: 280-284$

60. Gupta MA, Gupta AK. Quality of life of psoriasis patients. J Eur Acad Dermatol Venereol 2000;14:241-242

61. Kurd SK, Troxel AB, Crits-Christoph P, et al. The risk of depression, anxiety, and suicidality in patients with psoriasis. A population-based cohort study. Arch Dermatol 2010;146:891-895

62. Kimball AB, Jacobson C, Weiss $\mathrm{S}$, et al. The psychosocial burden of psoriasis. Am J Clin Dermatol 2005;6:383-392

63. de Arruda LH, De Moraes AP. The impact of psoriasis on quality of life. Br J Dermatol 2001;144: 33-36

64. Ciocon DH, Horn EJ, Kimball AB. Quality of life and treatment satisfaction among patients with psoriasis and psoriatic arthritis and patients with psoriasis only. Am J Clin Dermatol. 2008;9:111-117

65. Boers M, Kirwan JR, Wells G, et al. Developing core outcome measurement sets for clinical trials: OMERACT filter 2.0. J Clin Epidemiol. 2014;67:745-53 
66. http://oml.eular.org/ accessed on 2 December 2017

67. Boers M, Kirwan JR, Gossec L, et al. How to choose core outcome measurement sets for clinical trials: OMERACT 11 approves filter 2.0. J Rheumatol. $2014 ; 41: 1025-1030$

68. Castrejón I, Gossec L, Carmona L. The EULAR Outcome Measures Library: an evolutional database of validated patient-reported instruments. Ann Rheum Dis. $2015 ; 74: 475-476$.

69. Wells G, Li T, Maxwell L, et al. Responsiveness of patient reported outcomes including fatigue, sleep quality, activity limitation, and quality of life following treatment with abatacept for rheumatoid arthritis. Ann Rheum Dis. 2008;67:260-265. 70. Leung YY, Ho KW, Zhu TY, et al. Testing scaling assumptions, reliability and validity of medical outcomes study short-form 36 health survey in psoriatic arthritis. Rheumatology (Oxford). 2010 Aug;49(8):1495-501

71. Ware JE, Sherbourne CD. The RAND 36 Short-form Health Status Survey: Conceptual framework and item selection. Med Care 1992;30:473-481

72. Husted JA, Gladman DD, Farewell VT, et aş. Validating the SF-36 health questionnaire in patients with psoriatic arthritis. J Rheumatol 1997;24:511-517

73. EuroQol Group. EuroQol-a new facility for the measurement of health-related quality of life. Health Policy. 1990;16:199-208

74. Mease PJ. Measures of psoriatic arthritis: tender and swollen joint assessment, psoriasis area and severity index (PASI), nail psoriasis severity index (NAPSI), modified nail psoriasis severity index (mNAPSI), Mander/Newcastle enthesitis index (MEI), Leeds enthesitis index (LEI), Spondyloarthritis Research Consortium of 
Canada (SPARCC), Maastricht ankylosing spondylitis enthesis score (MASES), Leeds dactylitis index (LDI), patient global for psoriatic arthritis, dermatology life quality index (DLQI), psoriatic arthritis quality of life (PsAQOL), functional assessment of chronic illness therapy-fatigue (FACIT-F), psoriatic arthritis response criteria (PsARC), psoriatic arthritis joint activity index (PSAJAI), disease activity in psoriatic arthritis (DAPSA), and composite psoriatic disease activity index (CPDAI). Arthritis Care Res (Hoboken) 2011;63(Suppl 11):S64-85

75. Finlay AY, Khan GK. Dermatology Life Quality Index (DLQI)-a simple practical measure for routine clinical use. Clin Exp Dermatol. 1994;19:210-216

76. Feldman SR, Kimball AB, Krueger GG, et al. Etanercept improves the health-related quality of life of patients with psoriasis: results of a phase III randomized clinical trial. J Am Acad Dermatol. 2005;53:887-889

77. Højgaard P, Klokker L, Orbai AM, et al. A systematic review of measurement properties of patient reported outcome measures in psoriatic arthritis: A GRAPPAOMERACT initiative. Semin Arthritis Rheum. 2017; 9. pii: S0049-0172(17)30182-8

78. de Wit M, Kvien T, Gossec L. Patient participation as an integral part of patient reported outcomes development guarantees the representativeness of the patient voice - A case-study from the field of rheumatology. RMD Open 2015;1:1 e000129

79. http://www.eular.org/tools_products_.cfm, accessed on 2 December 2017

80. Di Carlo M, Becciolini A, Lato V, et al. The 12-item Psoriatic Arthritis Impact of Disease Questionnaire: Construct Validity, Reliability, and Interpretability in a Clinical Setting. J Rheumatol. 2017;44:279-285 
81. Holland R, Tillett W, Korendowych E, et al. Validation of the Psoriatic Arthritis Impact of Disease (PsAID) Questionnaire and its potential as a single-item outcome measure in clinical practice. Ann Rheum Dis. 2017 Nov 16 pii: annrheumdis-2017-211996. doi: 10.1136/annrheumdis-2017-211996. [Epub ahead of print]

82. Salaffi F, Di Carlo M, Carotti M, et al. The Psoriatic Arthritis Impact of Disease 12item questionnaire: equivalence, reliability, validity, and feasibility of the touch-screen administration versus the paper-and-pencil version. Ther Clin Risk Manag. $2016 ; 12: 631-642$

83. Healy PJ, Helliwell PS. Psoriatic arthritis quality of life instrument: an assessment of sensitivity and response to change. J Rheumatol. 2008;35:1359-1361

84. Gezer O, Batmaz I, Sariyildiz MA, et al. Sleep quality in patients with psoriatic arthritis. Int J Rheum Dis. 2017;20:1212-1218

85. Gladman D, Fleischmann R, Coteur G, et al. Effect of certolizumab pegol on multiple facets of psoriatic arthritis as reported by patients: 24-week patient-reported outcome results of a phase III, multicenter study. Arthritis Care Res (Hoboken). 2014;66:1085-1092

86. Taccari E, Spadaro A, Rinaldi $T$ et al. Comparison of the Health Assessment Questionnaire and Arthritis Impact Measurement Scale in patients with psoriatic arthritis. Rev Rhum Engl Ed. 1998;65:751-758

87. Coaccioli S, Bruno AA, Celi G, et al. Validation of an original questionnaire for patients with psoriatic arthritis: the Psoriatic Arthritis Impact Profile (PAIP). Clin Ter. 2014;165:e100-e108 
88. Chiricozzi A, Bianchi L, Zangrilli A et al. Quality of life of psoriatic patients evaluated by a new psychometric assessment tool: PsoDisk. European Journal of Dermatology. 2015;25:64-69

89. El MY, El GM, Youssef SS et al. Incorporating patient reported outcome measures in clinical practice: Development and validation of a questionnaire for inflammatory arthritis. Clinical and Experimental Rheumatology. 2010;28:734-744

90. Cohen JM, Halim K, Joyce CJ et al. Shedding Light on the "Hidden Psoriasis": A Pilot Study of the Inverse Psoriasis Burden of Disease (IPBOD) Questionnaire. J Drugs Dermatol. 2016;15:1011-6

91. Gossec L, Smolen JS, Ramiro S, et al. European League Against Rheumatism (EULAR) recommendations for the management of psoriatic arthritis with pharmacological therapies: 2015 update. Ann Rheum Dis. 2016;75:499-510.

92. Stoffer MA, Smolen JS, Woolf A, et al. Development of patient-centred standards of care for rheumatoid arthritis in Europe: the eumusc.net project. Ann Rheum Dis. 2014;73:902-905.

93. http://www.pcori.org/ accessed on 2 December 2017

94. Smolen JS, Landewé R, Breedveld FC, et al. EULAR recommendations for the management of rheumatoid arthritis with synthetic and biological disease-modifying antirheumatic drugs: 2013 update. Ann Rheum Dis. 2014;73:492-509.

95. Sanderson TC, Hewlett SE, Flurey C, et al. The impact triad (severity, importance, self-management) as a method of enhancing measurement of personal life impact of rheumatic diseases. J Rheumatol. 2011;38:191-194. 
96. Staley K. Summary Exploring Impact: Public involvement in NHS. Eastleigh: public health and social care research. INVOLVE, 2009. 